\title{
General nonconvex variational inclusions and fixed point problems
}

Narin Petrot ${ }^{1}$ and Javad Balooee ${ }^{2^{*}}$

\section{"Correspondence:}

javad.balooee@gmail.com

${ }^{2}$ Department of Mathematics, Sari

Branch, Islamic Azad University, Sari,

Iran

Full list of author information is

available at the end of the article

\begin{abstract}
In this paper, we introduce and study a new system of general nonconvex variational inclusions involving four different nonlinear operators (SGNVI) and prove the equivalence between the SGNVI and a fixed point problem. By using this equivalent formulation, we establish the existence and uniqueness theorem for solution of the SGNVI. We use the foregoing equivalent alternative formulation and two nearly uniformly Lipschitzian mappings $S_{1}$ and $S_{2}$ to suggest and analyze some new two-step projection iterative algorithms for finding an element of the set of fixed points of the nearly uniformly Lipschitzian mapping $\mathcal{Q}=\left(S_{1}, S_{2}\right)$, which is the unique solution of the SGNVI. Further, the convergence analysis of the suggested iterative algorithms under suitable conditions is studied.

MSC: Primary 47H05; secondary 47J20; 49J40; 90C33

Keywords: variational inclusions; fixed point problems; prox-regularity; nearly uniformly Lipschitzian mappings; two-step projection iterative algorithms; convergence analysis
\end{abstract}

\section{Introduction}

The variational principle, the origin of which can be traced back to Fermat, Newton, Leibniz, Bernoulli, Euler, and Lagrange, has been one of the major branches of mathematical and engineering sciences for more than two centuries. It can be used to interpret the basic principles of mathematical and physical sciences in the form of simplicity and elegance. The variational principles have played a fundamental and important part as unifying influence in sciences and have played a fundamental role in the development of general theory of relativity, gauge field theory in modern particle physics, and solution theory. In recent years, these variational principles have been enriched by the discovery of the variational inequalities theory, which is mainly due to Stampacchia [1] in 1964. The variational inequalities theory constituted a significant and novel extension of the variational principles and describe a broad spectrum of interesting and fascinating developments involving a link among various fields of mathematics, physics, economics, equilibrium, financial, optimization, regional, and engineering sciences. In fact, it has been shown that the variational inequalities theory provides the most natural, direct, simple, unified, and efficient framework for a general treatment of a wide class of problems. Many research papers have been written lately, both on the theory and applications of this field. Important connections with main areas of pure and applied sciences have been made, see for example [2-4] and the references cited therein. The development of variational inequality theory can be

(C) 2013 Petrot and Balooee; licensee Springer. This is an Open Access article distributed under the terms of the Creative Commons Attribution License (http://creativecommons.org/licenses/by/2.0), which permits unrestricted use, distribution, and reproduction in any medium, provided the original work is properly cited. 
viewed as the simultaneous pursuit of two different lines of research. On the one hand, it reveals the fundamental facts on the qualitative aspects of the solution to important classes of problems; on the other hand, it also enables us to develop highly efficient and powerful new numerical methods to solve, for example, obstacle, unilateral, free, moving and the complex equilibrium problems. One of the most interesting and important problems in variational inequality theory is the development of an efficient numerical method. There is a substantial number of numerical methods, including projection method and its variant forms, Wiener-Holf (normal) equations, auxiliary principle, and descent framework for solving variational inequalities and complementarity problems.

Projection method and its variant forms represent important tool for finding the approximate solution of various types of variational and quasi-variational inequalities, the origin of which can be traced back to Lions and Stampacchia [5]. The projection type methods were developed in 1970's and 1980's. The main idea in this technique is to establish the equivalence between the variational inequalities and the fixed point problems using the concept of projection. This alternative formulation enables us to suggest some iterative methods for computing the approximate solution.

Verma [6], Chang et al. [7] and Huang and Noor [8] introduced and studied systems of nonlinear variational inequalities, and by using the projection operator technique, they proposed some projection iterative algorithms for solving these systems of variational inequalities. They also studied the convergence analysis of the proposed iterative algorithms under some certain conditions.

It should be pointed out that almost all the results regarding the existence and iterative schemes for solving variational inequalities and related optimizations problems are being considered in the convexity setting. Consequently, all the techniques are based on the properties of the projection operator over convex sets, which may not hold in general, when the sets are nonconvex. It is known that the uniformly prox-regular sets are nonconvex and include the convex sets as special cases, for more details, see for example [9-12]. In recent years, Balooee et al. [13], Bounkhel et al. [9], Noor et al. [14], Pang et al. [15], Petrot [16] and Suwannawit et al. [17] have considered variational inequalities in the context of uniformly prox-regular sets.

On the other hand, related to the variational inequalities, we have the problem of finding the fixed points of nonexpansive mappings, which is the subject of current interest in functional analysis. It is natural to consider a unified approach to these two different problems. Motivated and inspired by the research going in this direction, Noor and Huang [18] considered the problem of finding a common element of the set of solutions of variational inequalities and the set of fixed points of nonexpansive mappings. It is well known that every nonexpansive mapping is a Lipschitzian mapping. Lipschitzian mappings have been generalized by various authors. Sahu [19] introduced and investigated nearly uniformly Lipschitzian mappings as a generalization of Lipschitzian mappings. In the recent past, some works in this direction for finding the solutions of the variational inequalities/inclusion problems and the fixed points of the nearly uniformly Lipschitzian mappings have been done, see, for example, $[13,20,21]$.

Motivated and inspired by the above works, in the present paper, we first introduce and consider a new system of general nonconvex variational inclusions involving four different nonlinear operators (SGNVI). We prove the equivalence between the SGNVI and a fixed point problem, and then by this equivalent formulation, we discuss the existence 
and uniqueness of solution of the SGNVI. By using two nearly uniformly Lipschitzian mappings $S_{1}$ and $S_{2}$ and the aforesaid equivalent alternative formulation, we suggest and analyze some new projection two-step iterative algorithms for finding an element of the set of fixed points of the nearly uniformly Lipschitzian mapping $\mathcal{Q}=\left(S_{1}, S_{2}\right)$, which is the unique solution of the SGNVI. The convergence analysis of the suggested iterative algorithms under suitable conditions is also studied.

\section{Formulations and basic facts}

Throughout this article, we let $\mathcal{H}$ be a real Hilbert space, which is equipped with an inner product $\langle\cdot, \cdot\rangle$ and the corresponding norm $\|\cdot\|$, and we let $K$ be a nonempty and closed subset of $\mathcal{H}$. We denote by $d_{K}(\cdot)$ or $d(\cdot, K)$ the usual distance function to the subset $K$, i.e., $d_{K}(u)=\inf _{v \in K}\|u-v\|$. Let us recall the following well-known definitions and some auxiliary results of nonlinear convex analysis and nonsmooth analysis [10-12, 22].

Definition 2.1 Let $u \in \mathcal{H}$ be a point not lying in $K$. A point $v \in K$ is called $a$ closest point or a projection of $u$ onto $K$ if $d_{K}(u)=\|u-v\|$. The set of all such closest points is denoted by $P_{K}(u)$, i.e.,

$$
P_{K}(u):=\left\{v \in K: d_{K}(u)=\|u-v\|\right\} .
$$

Definition 2.2 The proximal normal cone of $K$ at a point $u \in K$ is given by

$$
N_{K}^{P}(u):=\left\{\xi \in \mathcal{H}: \exists \alpha>0 \text { such that } u \in P_{K}(u+\alpha \xi)\right\} .
$$

Clarke et al. [10], in Proposition 1.1.5, give a characterization of $N_{K}^{P}(u)$ as follows.

Lemma 2.1 Let $K$ be a nonempty closed subset in $\mathcal{H}$. Then $\xi \in N_{K}^{P}(u)$ if and only if there exists a constant $\alpha=\alpha(\xi, u)>0$ such that $\langle\xi, v-u\rangle \leq \alpha\|v-u\|^{2}$ for all $v \in K$.

The above inequality is called the proximal normal inequality. The special case in which $K$ is closed and convex is an important one. In Proposition 1.1.10 of [10], the authors give the following characterization of the proximal normal cone for the closed and convex subset $K \subset \mathcal{H}$.

Lemma 2.2 Let $K$ be a nonempty, closed and convex subset in $\mathcal{H}$. Then $\xi \in N_{K}^{P}(u)$ if and only if $\langle\xi, v-u\rangle \leq 0$ for all $v \in K$.

Definition 2.3 Let $X$ be a real Banach space, and let $f: X \rightarrow \mathbb{R}$ be the Lipschitz with constant $\tau$ near a given point $x \in X$, that is, for some $\varepsilon>0$, we have $|f(y)-f(z)| \leq \tau\|y-z\|$ for all $y, z \in B(x ; \varepsilon)$, where $B(x ; \varepsilon)$ denotes the open ball of radius $\varepsilon>0$ and centered at $x$. The generalized directional derivative of $f$ at $x$ in the direction $v$, denoted as $f^{\circ}(x ; v)$, is defined as follows.

$$
f^{\circ}(x ; v)=\limsup _{y \rightarrow x, t \downarrow 0} \frac{f(y+t v)-f(y)}{t},
$$

where $y$ is a vector in $X$ and $t$ is a positive scalar. 
The generalized directional derivative defined earlier can be used to develop a notion of tangency that does not require $K$ to be smooth or convex.

Definition 2.4 The tangent cone $T_{K}(x)$ to $K$ at a point $x$ in $K$ is defined as follows:

$$
T_{K}(x):=\left\{v \in \mathcal{H}: d_{K}^{\circ}(x ; v)=0\right\}
$$

Having defined a tangent cone, the likely candidate for the normal cone is the one obtained from $T_{K}(x)$ by polarity. Accordingly, we define the normal cone of $K$ at $x$ by polarity with $T_{K}(x)$ as follows:

$$
N_{K}(x):=\left\{\xi:\langle\xi, v\rangle \leq 0, \forall v \in T_{K}(x)\right\}
$$

The Clarke normal cone, denoted by $N_{K}^{C}(x)$, is defined by $N_{K}^{C}(x):=\overline{c o}\left[N_{K}^{P}(x)\right]$, where $\overline{c o}[S]$ denotes the closure of the convex hull of $S$.

Clearly, $N_{K}^{P}(x) \subseteq N_{K}^{C}(x)$. Note that $N_{K}^{C}(x)$ is a closed and convex cone, whereas $N_{K}^{P}(x)$ is convex, but may not be closed. For further details on this topic, we refer to $[10,12,22]$ and the references therein.

In 1995, Clarke et al. [11] introduced and studied a new class of nonconvex sets, called proximally smooth sets; subsequently, Poliquin et al. in [12], investigated the aforementioned set under the name of uniformly prox-regular sets. These have been successfully used in many nonconvex applications in areas such as optimizations, economic models, dynamical systems, differential inclusions, etc. For such applications, see [23-26]. This class seems particularly well suited to overcome the difficulties, which arise due to the nonconvexity assumptions on $K$. We take the following characterization, proved in [11], as a definition of this class. We point out that the original definition was given in terms of the differentiability of the distance function, see [11].

Definition 2.5 For any $r \in(0,+\infty]$, a subset $K_{r}$ of $\mathcal{H}$ is called normalized uniformly proxregular (or uniformly $r$-prox-regular [11]) if every nonzero proximal normal to $K_{r}$ can be realized by an $r$-ball. This means that for all $\bar{x} \in K_{r}$ and all $0 \neq \xi \in N_{K_{r}}^{P}(\bar{x})$,

$$
\left\langle\frac{\xi}{\|\xi\|}, x-\bar{x}\right\rangle \leq \frac{1}{2 r}\|x-\bar{x}\|^{2}, \quad \forall x \in K_{r} .
$$

Obviously, the class of normalized uniformly prox-regular sets is sufficiently large to include the class of convex sets, $p$-convex sets, $C^{1,1}$ submanifolds (possibly with a boundary) of $\mathcal{H}$, the images under a $C^{1,1}$ diffeomorphism of convex sets and many other nonconvex sets, see $[11,27]$.

Lemma 2.3 [11] A closed set $K \subseteq \mathcal{H}$ is convex if and only if it is proximally smooth of radius $r$ for every $r>0$.

If $r=+\infty$, then, in view of Definition 2.5 and Lemma 2.3, the uniform $r$-prox-regularity of $K_{r}$ is equivalent to the convexity of $K_{r}$, which makes this class of great importance. For the case of that $r=+\infty$, we set $K_{r}=K$. 
The following proposition summarizes some important consequences of the uniform prox-regularity needed in the sequel.

Proposition 2.4 [11,12] Let $r>0$, and let $K_{r}$ be a nonempty closed and uniformly $r$-proxregular subset of $\mathcal{H}$. Let $U(r)=\left\{u \in \mathcal{H}: d_{K_{r}}(u)<r\right\}$. Then the following assertions hold

(a) For all $x \in U(r), P_{K_{r}}(x) \neq \emptyset$;

(b) For all $r^{\prime} \in(0, r), P_{K_{r}}$ is Lipschitz continuous with constant $\frac{r}{r-r^{\prime}}$ on $U\left(r^{\prime}\right)=\left\{u \in \mathcal{H}: d_{K_{r}}(u)<r^{\prime}\right\}$

(c) The proximal normal cone is closed as a set-valued mapping.

As a direct consequent of part (c) of Proposition 2.4, for any uniformly $r$-prox-regular subset $K_{r}$ of $\mathcal{H}$, we have $N_{K_{r}}^{C}(x)=N_{K_{r}}^{P}(x)$. Therefore, we define $N_{K_{r}}(x):=N_{K_{r}}^{C}(x)=N_{K_{r}}^{P}(x)$ for such a class of sets.

In order to make clear the concept of $r$-prox-regular sets, we state the following concrete example. The union of two disjoint intervals $[a, b]$ and $[c, d]$ is $r$-prox-regular with $r=\frac{c-b}{2}$, see $[9,10,12]$. The finite union of disjoint intervals is also $r$-prox-regular, and $r$ depends on the distances between the intervals.

\section{System of general nonconvex variational inclusions}

This section is concerned with the introduction of a new system of nonconvex variational inclusions and establishing of the existence and uniqueness theorem for solution of the aforesaid system.

Let $K_{r}$ be a uniformly $r$-prox-regular subset of $\mathcal{H}$, and let $T_{i}: \mathcal{H} \times \mathcal{H} \rightarrow \mathcal{H}$ and $g_{i}: \mathcal{H} \rightarrow$ $\mathcal{H}(i=1,2)$, be four nonlinear single-valued operators. For any given constants $\rho, \eta>0$, we consider the problem of finding $\left(x^{*}, y^{*}\right) \in K_{r} \times K_{r}$ such that

$$
\left\{\begin{array}{l}
g_{1}\left(y^{*}\right)-x^{*}-\rho T_{1}\left(y^{*}, x^{*}\right) \in N_{K_{r}}^{P}\left(x^{*}\right) \\
g_{2}\left(x^{*}\right)-y^{*}-\eta T_{2}\left(x^{*}, y^{*}\right) \in N_{K_{r}}^{P}\left(y^{*}\right)
\end{array}\right.
$$

which is called a system of general nonconvex variational inclusions involving four different nonlinear operators (SGNVI).

If for each $i=1,2, T_{i}=T: K_{r} \rightarrow \mathcal{H}$, is a univariate nonlinear operator and $g_{i} \equiv I$, the identity operator, then system (3.1) collapses to the system of finding $\left(x^{*}, y^{*}\right) \in K_{r} \times K_{r}$ such that

$$
\left\{\begin{array}{l}
y^{*}-x^{*}-\rho T\left(y^{*}\right) \in N_{K_{r}}^{P}\left(x^{*}\right), \\
x^{*}-y^{*}-\eta T\left(x^{*}\right) \in N_{K_{r}}^{P}\left(y^{*}\right),
\end{array}\right.
$$

which was considered and studied by Moudafi [28] and Petrot [16].

If $r=\infty$, i.e., $K_{r}=K$, the convex set in $\mathcal{H}$, and for each $i=1,2, g_{i}(\mathcal{H})=K$, then it follows from Lemma 2.2 that $\left(x^{*}, y^{*}\right) \in K \times K$ is a solution of system (3.1) if and only if

$$
\begin{cases}\left\langle\rho T_{1}\left(y^{*}, x^{*}\right)+x^{*}-g_{1}\left(y^{*}\right), g_{1}(x)-x^{*}\right\rangle \geq 0, & \forall x \in K, \\ \left\langle\eta T_{2}\left(x^{*}, y^{*}\right)+y^{*}-g_{2}\left(x^{*}\right), g_{2}(x)-y^{*}\right\rangle \geq 0, & \forall x \in K .\end{cases}
$$


Problem (3.3) is called a system of general nonlinear variational inequalities in the sense of convex analysis.

If $r=\infty$ and for each $i=1,2, g_{i} \equiv I$, then system (3.3) collapses to the system of finding $\left(x^{*}, y^{*}\right) \in K \times K$ such that

$$
\begin{cases}\left\langle\rho T_{1}\left(y^{*}, x^{*}\right)+x^{*}-y^{*}, x-x^{*}\right\rangle \geq 0, & \forall x \in K, \\ \left\langle\eta T_{2}\left(x^{*}, y^{*}\right)+y^{*}-x^{*}, x-y^{*}\right\rangle \geq 0, & \forall x \in K,\end{cases}
$$

which was introduced and studied by Huang and Noor [8].

Some special cases of system (3.4) are introduced and studied by Chang et al. [7], Verma $[6,29,30]$ and Stampacchia [1].

Now, we establish the existence and uniqueness of solution for SGNVI (3.1). For this end, we need the following lemma, in which the equivalence between SGNVI (3.1) and a fixed point problem is proved.

Lemma 3.1 Let $T_{i}, g_{i}(i=1,2), \rho$ and $\eta$ be the same as in SGNVI (3.1) such that for each $i=1,2, g_{i}(\mathcal{H})=K_{r}$. Then $\left(x^{*}, y^{*}\right) \in K_{r} \times K_{r}$ is a solution of system (3.1) if and only if

$$
\left\{\begin{array}{l}
x^{*}=P_{K_{r}}\left(g_{1}\left(y^{*}\right)-\rho T_{1}\left(y^{*}, x^{*}\right)\right), \\
y^{*}=P_{K_{r}}\left(g_{2}\left(x^{*}\right)-\eta T_{2}\left(x^{*}, y^{*}\right)\right),
\end{array}\right.
$$

provided that $\rho<\frac{r^{\prime}}{1+\left\|T_{1}\left(y^{*}, x^{*}\right)\right\|}$ and $\eta<\frac{r^{\prime}}{1+\left\|T_{2}\left(x^{*}, y^{*}\right)\right\|}$, for some $r^{\prime} \in(0, r)$, where $P_{K_{r}}$ is the projection of $\mathcal{H}$ onto $K_{r}$.

Proof Let $\left(x^{*}, y^{*}\right) \in K_{r} \times K_{r}$ be a solution of SGNVI (3.1). Since $g_{1}\left(y^{*}\right), g_{2}\left(x^{*}\right) \in K_{r}, \rho<$ $\frac{r^{\prime}}{1+\left\|T_{1}\left(y^{*}, x^{*}\right)\right\|}$ and $\eta<\frac{r^{\prime}}{1+\left\|T_{2}\left(x^{*}, y^{*}\right)\right\|}$, for some $r^{\prime} \in(0, r)$, it is easy to check that the two points $g_{1}\left(y^{*}\right)-\rho T_{1}\left(y^{*}, x^{*}\right)$ and $g_{2}\left(x^{*}\right)-\eta T_{2}\left(x^{*}, y^{*}\right)$ belong to $U\left(r^{\prime}\right)$. Therefore, the $r$-prox regularity of $K_{r}$ implies that the two sets $P_{K_{r}}\left(g_{1}\left(y^{*}\right)-\rho T_{1}\left(y^{*}, x^{*}\right)\right)$ and $P_{K_{r}}\left(g_{2}\left(x^{*}\right)-\eta T_{2}\left(x^{*}, y^{*}\right)\right)$ are nonempty and singleton, that is, equations (3.5) are well defined. Then, we have

$$
\begin{aligned}
& \left\{\begin{array}{l}
g_{1}\left(y^{*}\right)-x^{*}-\rho T_{1}\left(y^{*}, x^{*}\right) \in N_{K_{r}}^{P}\left(x^{*}\right), \\
g_{2}\left(x^{*}\right)-y^{*}-\eta T_{2}\left(x^{*}, y^{*}\right) \in N_{K_{r}}^{P}\left(y^{*}\right),
\end{array}\right. \\
& \Longleftrightarrow\left\{\begin{array}{l}
g_{1}\left(y^{*}\right)-\rho T_{1}\left(y^{*}, x^{*}\right) \in\left(I+N_{K_{r}}^{P}\right)\left(x^{*}\right), \\
g_{2}\left(x^{*}\right)-\eta T_{2}\left(x^{*}, y^{*}\right) \in\left(I+N_{K_{r}}^{P}\right)\left(y^{*}\right),
\end{array}\right. \\
& \Longleftrightarrow\left\{\begin{array}{l}
x^{*}=P_{K_{r}}\left(g_{1}\left(y^{*}\right)-\rho T_{1}\left(y^{*}, x^{*}\right)\right), \\
y^{*}=P_{K_{r}}\left(g_{2}\left(x^{*}\right)-\eta T_{2}\left(x^{*}, y^{*}\right)\right),
\end{array}\right.
\end{aligned}
$$

where $I$ is the identity operator, and we have used the well-known fact that $P_{K_{r}}=(I+$ $\left.N_{K_{r}}^{P}\right)^{-1}$.

Definition 3.1 A single-valued operator $T: \mathcal{H} \rightarrow \mathcal{H}$ is called 
(a) monotone if

$$
\langle T(x)-T(y), x-y\rangle \geq 0, \quad \forall x, y \in \mathcal{H}
$$

(b) $r$-strongly monotone if there exists a constant $r>0$ such that

$$
\langle T(x)-T(y), x-y\rangle \geq r\|x-y\|^{2}, \quad \forall x, y \in \mathcal{H}
$$

(c) $\gamma$-Lipschitz continuous if there exists a constant $\gamma>0$ such that

$$
\|T(x)-T(y)\| \leq \gamma\|x-y\|, \quad \forall x, y \in \mathcal{H}
$$

Theorem 3.2 Let $T_{i}, g_{i}(i=1,2), \rho$ and $\eta$ be the same as in system (3.1) such that for each $i=$ $1,2, g_{i}(\mathcal{H})=K_{r}$. Suppose further that for each $i=1,2$, the operator $T_{i}$ is $\xi_{i}$-strongly monotone and $v_{i}$-Lipschitz continuous in the first variable, $g_{i}$ is $\kappa_{i}$-strongly monotone and $\theta_{i}$-Lipschitz continuous. If the constants $\rho, \eta>0$ satisfy the following conditions:

$$
\rho<\frac{r^{\prime}}{1+\left\|T_{1}(y, x)\right\|} \quad \text { and } \quad \eta<\frac{r^{\prime}}{1+\left\|T_{2}(x, y)\right\|} \text {, }
$$

for some $r^{\prime} \in(0, r)$ and for all $x, y \in \mathcal{H}$, and

$$
\left\{\begin{array}{l}
\left|\rho-\frac{\xi_{1}}{v_{1}^{2}}\right|<\frac{\sqrt{r^{2} \xi_{1}^{2}-v_{1}^{2}\left(r^{2}-\left(r-r^{\prime}\right)^{2}\left(1-\mu_{1}\right)^{2}\right)}}{r v_{1}^{2}} \\
\left|\eta-\frac{\xi_{2}}{v_{2}^{2}}\right|<\frac{\sqrt{r^{2} \xi_{2}^{2}-v_{2}^{2}\left(r^{2}-\left(r-r^{\prime}\right)^{2}\left(1-\mu_{2}\right)^{2}\right)}}{r v_{2}^{2}} \\
r \xi_{i}>v_{i} \sqrt{r^{2}-\left(r-r^{\prime}\right)^{2}\left(1-\mu_{i}\right)^{2}}, \\
\mu_{i}=\frac{r}{r-r^{\prime}} \sqrt{1-\left(2 \kappa_{i}-\theta_{i}^{2}\right)}<1, \\
2 \kappa_{i}<1+\theta_{i}^{2} \quad(i=1,2),
\end{array}\right.
$$

then SGNVI (3.1) admits a unique solution.

Proof Define $\Psi, \Phi: \mathcal{H} \times \mathcal{H} \rightarrow K_{r}$ by

$$
\begin{aligned}
& \Psi(x, y)=P_{K_{r}}\left(g_{1}(y)-\rho T_{1}(y, x)\right), \\
& \Phi(x, y)=P_{K_{r}}\left(g_{2}(x)-\eta T_{2}(x, y)\right),
\end{aligned}
$$

for all $(x, y) \in \mathcal{H} \times \mathcal{H}$. Since $g_{1}(y), g_{2}(x) \in K_{r}$, it follows from condition (3.6) that the mappings $\Psi$ and $\Phi$ are well defined. Define $\|\cdot\|_{*}$ on $\mathcal{H} \times \mathcal{H}$ by

$$
\|(x, y)\|_{*}=\|x\|+\|y\|, \quad \forall(x, y) \in \mathcal{H} \times \mathcal{H} .
$$

It is clear that $\left(\mathcal{H} \times \mathcal{H},\|\cdot\|_{*}\right)$ is a Banach space. Also, define $F: \mathcal{H} \times \mathcal{H} \rightarrow K_{r} \times K_{r} \subseteq \mathcal{H} \times \mathcal{H}$ as below:

$$
F(x, y)=(\Psi(x, y), \Phi(x, y)), \quad \forall(x, y) \in \mathcal{H} \times \mathcal{H}
$$


We show that the mapping $F$ is contraction. Let $(x, y),(\hat{x}, \hat{y}) \in \mathcal{H} \times \mathcal{H}$ be given. Applying Proposition 2.4, we have

$$
\begin{aligned}
& \|\Psi(x, y)-\Psi(\hat{x}, \hat{y})\| \\
& \quad=\left\|P_{K_{r}}\left(g_{1}(y)-\rho T_{1}(y, x)\right)-P_{K_{r}}\left(g_{1}(\hat{y})-\rho T_{1}(\hat{y}, \hat{x})\right)\right\| \\
& \quad \leq \frac{r}{r-r^{\prime}}\left\|g_{1}(y)-g_{1}(\hat{y})-\rho\left(T_{1}(y, x)-T_{1}(\hat{y}, \hat{x})\right)\right\| \\
& \quad \leq \frac{r}{r-r^{\prime}}\left(\left\|y-\hat{y}-\left(g_{1}(y)-g_{1}(\hat{y})\right)\right\|+\left\|y-\hat{y}-\rho\left(T_{1}(y, x)-T_{1}(\hat{y}, \hat{x})\right)\right\|\right) .
\end{aligned}
$$

From $\kappa_{1}$-strongly monotonicity and $\theta_{1}$-Lipschitz continuity of $g_{1}$, it follows that

$$
\begin{aligned}
\left\|y-\hat{y}-\left(g_{1}(y)-g_{1}(\hat{y})\right)\right\|^{2} & =\|y-\hat{y}\|^{2}-2\left\langle g_{1}(y)-g_{1}(\hat{y}), y-\hat{y}\right\rangle+\left\|g_{1}(y)-g_{1}(\hat{y})\right\|^{2} \\
& \leq\left(1-2 \kappa_{1}\right)\|y-\hat{y}\|^{2}+\left\|g_{1}(y)-g_{1}(\hat{y})\right\|^{2} \\
& \leq\left(1-2 \kappa_{1}+\theta_{1}^{2}\right)\|y-\hat{y}\|^{2} .
\end{aligned}
$$

Since $T_{1}$ is $\xi_{1}$-strongly monotone and $v_{1}$-Lipschitz continuous in the first variable, we get

$$
\begin{aligned}
\| y & -\hat{y}-\rho\left(T_{1}(y, x)-T_{1}(\hat{y}, \hat{x})\right) \|^{2} \\
& =\|y-\hat{y}\|^{2}-2 \rho\left(T_{1}(y, x)-T_{1}(\hat{y}, \hat{x}), y-\hat{y}\right)+\rho^{2}\left\|T_{1}(y, x)-T_{1}(\hat{y}, \hat{x})\right\|^{2} \\
& \leq\left(1-2 \rho \xi_{1}\right)\|y-\hat{y}\|^{2}+\rho^{2}\left\|T_{1}(y, x)-T_{1}(\hat{y}, \hat{x})\right\|^{2} \\
& \leq\left(1-2 \rho \xi_{1}+\rho^{2} v_{1}^{2}\right)\|y-\hat{y}\|^{2} .
\end{aligned}
$$

Substituting (3.11) and (3.12) in (3.10), we obtain

$$
\|\Psi(x, y)-\Psi(\hat{x}, \hat{y})\| \leq \frac{r}{r-r^{\prime}}\left(\sqrt{1-2 \kappa_{1}+\theta_{1}^{2}}+\sqrt{1-2 \rho \xi_{1}+\rho^{2} v_{1}^{2}}\right)\|y-\hat{y}\| .
$$

Like in the proofs of (3.10)-(3.13), one can obtain

$$
\|\Phi(x, y)-\Phi(\hat{x}, \hat{y})\| \leq \frac{r}{r-r^{\prime}}\left(\sqrt{1-2 \kappa_{2}+\theta_{2}^{2}}+\sqrt{1-2 \eta \xi_{2}+\eta^{2} v_{2}^{2}}\right)\|x-\hat{x}\| .
$$

Applying (3.13) and (3.14), we have

$$
\|\Psi(x, y)-\Psi(\hat{x}, \hat{y})\|+\|\Phi(x, y)-\Phi(\hat{x}, \hat{y})\| \leq \varphi\|x-\hat{x}\|+\psi\|y-\hat{y}\|,
$$

where

$$
\begin{aligned}
& \psi=\frac{r}{r-r^{\prime}}\left(\sqrt{1-2 \kappa_{1}+\theta_{1}^{2}}+\sqrt{1-2 \rho \xi_{1}+\rho^{2} v_{1}^{2}}\right), \\
& \varphi=\frac{r}{r-r^{\prime}}\left(\sqrt{1-2 \kappa_{2}+\theta_{2}^{2}}+\sqrt{1-2 \eta \xi_{2}+\eta^{2} v_{2}^{2}}\right) .
\end{aligned}
$$

It follows from (3.9) and (3.15) that

$$
\|F(x, y)-F(\hat{x}, \hat{y})\|_{*} \leq \vartheta\|(x, y)-(\hat{x}, \hat{y})\|_{*}
$$


where $\vartheta=\max \{\varphi, \psi\}$. Condition (3.7) implies that $0 \leq \vartheta<1$, and so, (3.16) guarantees that the mapping $F$ is contraction. According to the Banach fixed point theorem, there exists a unique point $\left(x^{*}, y^{*}\right) \in \mathcal{H} \times \mathcal{H}$ such that $F\left(x^{*}, y^{*}\right)=\left(x^{*}, y^{*}\right)$. It follows from (3.8) and (3.9) that $x^{*}=P_{K_{r}}\left(g_{1}\left(y^{*}\right)-\rho T_{1}\left(y^{*}, x^{*}\right)\right)$ and $y^{*}=P_{K_{r}}\left(g_{2}\left(x^{*}\right)-\eta T_{2}\left(x^{*}, y^{*}\right)\right)$. Now, from Lemma 3.1, it follows that $\left(x^{*}, y^{*}\right) \in K_{r} \times K_{r}$ is a unique solution of SGNVI (3.1), and this completes the proof.

Theorem 3.3 Let $T_{i}, g_{i}(i=1,2), \rho$ and $\eta$ be the same as in system (3.3) such that for each $i=1,2$, the operator $T_{i}$ is $\xi_{i}$-strongly monotone and $v_{i}$-Lipschitz continuous in the first variable, $g_{i}$ is $\kappa_{i}$-strongly monotone and $\theta_{i}$-Lipschitz continuous. If the constants $\rho, \eta>0$ satisfy the following conditions:

$$
\left\{\begin{array}{l}
\left|\rho-\frac{\xi_{1}}{v_{1}^{2}}\right|<\frac{\sqrt{\xi_{1}^{2}-v_{1}^{2} \mu_{1}\left(2-\mu_{1}\right)}}{v_{1}^{2}} \\
\left|\eta-\frac{\xi_{2}}{v_{2}^{2}}\right|<\frac{\sqrt{\xi_{2}^{2}-v_{2}^{2} \mu_{2}\left(2-\mu_{2}\right)}}{v_{2}^{2}} \\
\xi_{i}>v_{i} \sqrt{\mu_{i}\left(2-\mu_{i}\right)} \quad(i=1,2), \\
\mu_{i}=\sqrt{1-\left(2 \kappa_{i}-\theta_{i}^{2}\right)}<1 \quad(i=1,2), \\
2 \kappa_{i}<1+\theta_{i}^{2} \quad(i=1,2)
\end{array}\right.
$$

then system (3.3) admits a unique solution.

\section{Iterative algorithms and convergence analysis}

We need to recall that a nonlinear mapping $T: \mathcal{H} \rightarrow \mathcal{H}$ is called nonexpansive if $\| T x-$ $T y\|\leq\| x-y \|$ for all $x, y \in \mathcal{H}$. In recent years, nonexpansive mappings have been generalized and investigated by various authors. One of these generalizations is the class of nearly uniformly Lipschitzian mappings. In this section, we first recall several generalizations of nonexpansive mappings, which have been introduced in recent years. Then, we use two nearly uniformly Lipschitzian mappings $S_{1}$ and $S_{2}$ and the equivalent alternative formulation (3.5) to suggest and analyze some new two-step projection iterative algorithms for finding an element of the set of fixed points $\mathcal{Q}=\left(S_{1}, S_{2}\right)$, which is the unique solution of SGNVI (3.1). In the next definitions, several generalizations of nonexpansive mappings are stated.

Definition 4.1 A nonlinear mapping $T: \mathcal{H} \rightarrow \mathcal{H}$ is called

(a) L-Lipschitzian if there exists a constant $L>0$ such that

$$
\|T x-T y\| \leq L\|x-y\|, \quad \forall x, y \in \mathcal{H}
$$

(b) generalized Lipschitzian if there exists a constant $L>0$ such that

$$
\|T x-T y\| \leq L(\|x-y\|+1), \quad \forall x, y \in \mathcal{H}
$$

(c) generalized (L,M)-Lipschitzian [19] if there exist two constants $L, M>0$ such that

$$
\|T x-T y\| \leq L(\|x-y\|+M), \quad \forall x, y \in \mathcal{H}
$$


(d) asymptotically nonexpansive [31] if there exists a sequence $\left\{k_{n}\right\} \subseteq[1, \infty)$ with $\lim _{n \rightarrow \infty} k_{n}=1$ such that for each $n \in \mathbb{N}$,

$$
\left\|T^{n} x-T^{n} y\right\| \leq k_{n}\|x-y\|, \quad \forall x, y \in \mathcal{H}
$$

(e) pointwise asymptotically nonexpansive [32] if, for each integer $n \geq 1$,

$$
\left\|T^{n} x-T^{n} y\right\| \leq \alpha_{n}(x)\|x-y\|, \quad x, y \in \mathcal{H},
$$

where $\alpha_{n} \rightarrow 1$ pointwise on $X$;

(f) uniformly L-Lipschitzian if there exists a constant $L>0$ such that for each $n \in \mathbb{N}$,

$$
\left\|T^{n} x-T^{n} y\right\| \leq L\|x-y\|, \quad \forall x, y \in \mathcal{H}
$$

Definition 4.2 [19] A nonlinear mapping $T: \mathcal{H} \rightarrow \mathcal{H}$ is said to be

(a) nearly Lipschitzian with respect to the sequence $\left\{a_{n}\right\}$ if for each $n \in \mathbb{N}$, there exists a constant $k_{n}>0$ such that

$$
\left\|T^{n} x-T^{n} y\right\| \leq k_{n}\left(\|x-y\|+a_{n}\right), \quad \forall x, y \in \mathcal{H},
$$

where $\left\{a_{n}\right\}$ is a fix sequence in $[0, \infty)$ with $a_{n} \rightarrow 0$, as $n \rightarrow \infty$.

For an arbitrary, but fixed $n \in \mathbb{N}$, the infimum of constants $k_{n}$ in (4.1) is called nearly Lipschitz constant and is denoted by $\eta\left(T^{n}\right)$. Notice that

$$
\eta\left(T^{n}\right)=\sup \left\{\frac{\left\|T^{n} x-T^{n} y\right\|}{\|x-y\|+a_{n}}: x, y \in \mathcal{H}, x \neq y\right\} .
$$

A nearly Lipschitzian mapping $T$ with the sequence $\left\{\left(a_{n}, \eta\left(T^{n}\right)\right)\right\}$ is said to be

(b) nearly nonexpansive if $\eta\left(T^{n}\right)=1$ for all $n \in \mathbb{N}$, that is,

$$
\left\|T^{n} x-T^{n} y\right\| \leq\|x-y\|+a_{n}, \quad \forall x, y \in \mathcal{H}
$$

(c) nearly asymptotically nonexpansive if $\eta\left(T^{n}\right) \geq 1$ for all $n \in \mathbb{N}$ and $\lim _{n \rightarrow \infty} \eta\left(T^{n}\right)=1$, in other words, $k_{n} \geq 1$ for all $n \in \mathbb{N}$ with $\lim _{n \rightarrow \infty} k_{n}=1$;

(d) nearly uniformly L-Lipschitzian if $\eta\left(T^{n}\right) \leq L$ for all $n \in \mathbb{N}$, in other words, $k_{n}=L$ for all $n \in \mathbb{N}$.

Remark 4.1 It should be pointed out that

(a) Every nonexpansive mapping is an asymptotically nonexpansive mapping, and every asymptotically nonexpansive mapping is a pointwise asymptotically nonexpansive mapping. Also, the class of Lipschitzian mappings properly includes the class of pointwise asymptotically nonexpansive mappings.

(b) It is obvious that every Lipschitzian mapping is a generalized Lipschitzian mapping. Furthermore, every mapping with a bounded range is a generalized Lipschitzian mapping. It is easy to see that the class of generalized $(L, M)$-Lipschitzian mappings is more general than the class of generalized Lipschitzian mappings. 
(c) Clearly, the class of nearly uniformly $L$-Lipschitzian mappings properly includes the class of generalized $(L, M)$-Lipschitzian mappings and that of uniformly $L$-Lipschitzian mappings. Note that every nearly asymptotically nonexpansive mapping is nearly uniformly $L$-Lipschitzian.

Some interesting examples to investigate relations between these mappings, introduced in Definitions 4.1 and 4.2, can be found in [13].

Let $S_{1}: K_{r} \rightarrow K_{r}$ be a nearly uniformly $L_{1}$-Lipschitzian mapping with the sequence $\left\{a_{n}\right\}_{n=0}^{\infty}$, and let $S_{2}: K_{r} \rightarrow K_{r}$ be a nearly uniformly $L_{2}$-Lipschitzian mapping with the sequence $\left\{b_{n}\right\}_{n=0}^{\infty}$. We define the self-mapping $\mathcal{Q}$ of $K_{r} \times K_{r}$ as follows:

$$
\mathcal{Q}(x, y)=\left(S_{1} x, S_{2} y\right), \quad \forall x, y \in K_{r} .
$$

Then $\mathcal{Q}=\left(S_{1}, S_{2}\right): K_{r} \times K_{r} \rightarrow K_{r} \times K_{r}$ is a nearly uniformly $\max \left\{L_{1}, L_{2}\right\}$-Lipschitzian mapping with the sequence $\left\{a_{n}+b_{n}\right\}_{n=0}^{\infty}$ with respect to the norm $\|\cdot\|_{*}$ in $\mathcal{H} \times \mathcal{H}$. Because, for any $(x, y),\left(x^{\prime}, y^{\prime}\right) \in K_{r} \times K_{r}$ and $n \in \mathbb{N}$, we have

$$
\begin{aligned}
& \left\|\mathcal{Q}^{n}(x, y)-\mathcal{Q}^{n}\left(x^{\prime}, y^{\prime}\right)\right\|_{*} \\
& \quad=\left\|\left(S_{1}^{n} x, S_{2}^{n} y\right)-\left(S_{1}^{n} x^{\prime}, S_{2}^{n} y^{\prime}\right)\right\|_{*}=\left\|\left(S_{1}^{n} x-S_{1}^{n} x^{\prime}, S_{2}^{n} y-S_{2}^{n} y^{\prime}\right)\right\|_{*} \\
& \quad=\left\|S_{1}^{n} x-S_{1}^{n} x^{\prime}\right\|+\left\|S_{2}^{n} y-S_{2}^{n} y^{\prime}\right\| \leq L_{1}\left(\left\|x-x^{\prime}\right\|+a_{n}\right)+L_{2}\left(\left\|y-y^{\prime}\right\|+b_{n}\right) \\
& \quad \leq \max \left\{L_{1}, L_{2}\right\}\left(\left\|x-x^{\prime}\right\|+\left\|y-y^{\prime}\right\|+a_{n}+b_{n}\right) \\
& \quad=\max \left\{L_{1}, L_{2}\right\}\left(\left\|(x, y)-\left(x^{\prime}, y^{\prime}\right)\right\|_{*}+a_{n}+b_{n}\right) .
\end{aligned}
$$

We denote the sets of all fixed points of $S_{i}(i=1,2)$ and $\mathcal{Q}$ by $\operatorname{Fix}\left(S_{i}\right)$ and $\operatorname{Fix}(\mathcal{Q})$, respectively, and the set of all solutions of system (3.1) by $\operatorname{SGNVI}\left(K_{r}, T_{i}, g_{i}, i=1,2\right)$. In view of (4.2), for any $(x, y) \in K_{r} \times K_{r},(x, y) \in \operatorname{Fix}(\mathcal{Q})$ if and only if $x \in \operatorname{Fix}\left(S_{1}\right)$ and $y \in \operatorname{Fix}\left(S_{2}\right)$, that is, $\operatorname{Fix}(\mathcal{Q})=\operatorname{Fix}\left(S_{1}, S_{2}\right)=\operatorname{Fix}\left(S_{1}\right) \times \operatorname{Fix}\left(S_{2}\right)$. We now characterize the problem. Let the operators $T_{i}, g_{i}(i=1,2)$, and the constants $\rho, \eta$ be the same as in system (3.1). If $\left(x^{*}, y^{*}\right) \in \operatorname{Fix}(\mathcal{Q}) \cap \operatorname{SGNVI}\left(K_{r}, T_{i}, g_{i}, i=1,2\right), \rho<\frac{r^{\prime}}{1+\left\|T_{1}\left(y^{*}, x^{*}\right)\right\|}$ and $\eta<\frac{r^{\prime}}{1+\left\|T_{2}\left(x^{*}, y^{*}\right)\right\|}$, for some $r^{\prime} \in(0, r)$, then by using Lemma 3.1, it is easy to see that for each $n \in \mathbb{N} \cup\{0\}$,

$$
\left\{\begin{array}{l}
x^{*}=S_{1}^{n} x^{*}=P_{K_{r}}\left(g_{1}\left(y^{*}\right)-\rho T_{1}\left(y^{*}, x^{*}\right)\right)=S_{1}^{n} P_{K_{r}}\left(g_{1}\left(y^{*}\right)-\rho T_{1}\left(y^{*}, x^{*}\right)\right), \\
y^{*}=S_{2}^{n} y^{*}=P_{K_{r}}\left(g_{2}\left(x^{*}\right)-\eta T_{2}\left(x^{*}, y^{*}\right)\right)=S_{2}^{n} P_{K_{r}}\left(g_{2}\left(x^{*}\right)-\eta T_{2}\left(x^{*}, y^{*}\right)\right) .
\end{array}\right.
$$

The fixed point formulation (4.3) enables us to suggest the following iterative algorithm for finding an element of the set of fixed points of the nearly uniformly Lipschitzian mapping $\mathcal{Q}=\left(S_{1}, S_{2}\right)$, which is the unique solution of SGNVI (3.1).

Algorithm 4.2 Let $T_{i}, g_{i}(i=1,2), \rho$ and $\eta$ be the same as in SGNVI (3.1), and let the constants $\rho, \eta$ satisfy condition (3.6). For an arbitrary chosen initial point $\left(x_{0}, y_{0}\right) \in \mathcal{H} \times \mathcal{H}$, compute the iterative sequence $\left\{\left(x_{n}, y_{n}\right)\right\}_{n=0}^{\infty}$ in $\mathcal{H} \times \mathcal{H}$ in the following way:

$$
\left\{\begin{array}{l}
x_{n+1}=\left(1-\alpha_{n}\right) x_{n}+\alpha_{n} S_{1}^{n} P_{K_{r}}\left(g_{1}\left(y_{n}\right)-\rho T_{1}\left(y_{n}, x_{n}\right)\right), \\
y_{n+1}=\left(1-\alpha_{n}\right) y_{n}+\alpha_{n} S_{2}^{n} P_{K_{r}}\left(g_{2}\left(x_{n}\right)-\eta T_{2}\left(x_{n}, y_{n}\right)\right)
\end{array}\right.
$$


where $S_{1}, S_{2}: K_{r} \rightarrow K_{r}$ are two nearly uniformly Lipschitzian mappings, and $\left\{\alpha_{n}\right\}_{n=0}^{\infty}$ is a sequence in the interval $[0,1]$ such that $\sum_{n=1}^{\infty} \alpha_{n}=\infty$.

If for each $i=1,2, S_{i} \equiv I$, then Algorithm 4.2 reduces to the following algorithm.

Algorithm 4.3 Let $T_{i}, g_{i}(i=1,2), \rho$ and $\eta$ be the same as in SGNVI (3.1), and let the constants $\rho, \eta$ satisfy condition (3.6). For an arbitrary chosen initial point $\left(x_{0}, y_{0}\right) \in \mathcal{H} \times \mathcal{H}$, compute the iterative sequence $\left\{\left(x_{n}, y_{n}\right)\right\}_{n=0}^{\infty}$ in $\mathcal{H} \times \mathcal{H}$ in the following way:

$$
\left\{\begin{array}{l}
x_{n+1}=\left(1-\alpha_{n}\right) x_{n}+\alpha_{n} P_{K_{r}}\left(g_{1}\left(y_{n}\right)-\rho T_{1}\left(y_{n}, x_{n}\right)\right), \\
y_{n+1}=\left(1-\alpha_{n}\right) y_{n}+\alpha_{n} P_{K_{r}}\left(g_{2}\left(x_{n}\right)-\eta T_{2}\left(x_{n}, y_{n}\right)\right),
\end{array}\right.
$$

where the sequence $\left\{\alpha_{n}\right\}_{n=0}^{\infty}$ is the same as in Algorithm 4.2.

Now, we discuss the convergence analysis of iterative sequence generated by the projection iterative Algorithm 4.2. We need the following lemma for verifying our main results.

Lemma 4.4 [33] Let $\left\{a_{n}\right\}$ be a nonnegative real sequence, and let $\left\{b_{n}\right\}$ be a real sequence in $[0,1]$ such that $\sum_{n=0}^{\infty} b_{n}=\infty$. If there exists a positive integer $n_{0}$ such that

$$
a_{n+1} \leq\left(1-b_{n}\right) a_{n}+b_{n} c_{n}, \quad \forall n \geq n_{0},
$$

where $c_{n} \geq 0$ for all $n \geq 0$ and $\lim _{n \rightarrow \infty} c_{n}=0$, then $\lim _{n \rightarrow 0} a_{n}=0$.

Theorem 4.5 Let $T_{i}, g_{i}(i=1,2), \rho$ and $\eta$ be the same as in Theorem 3.2. Assume that all the conditions of Theorem 3.2 hold and the constants $\rho$, $\eta$ satisfy condition (3.6). Suppose that $S_{1}: K_{r} \rightarrow K_{r}$ is a nearly uniformly $L_{1}$-Lipschitzian mapping with the sequence $\left\{b_{n}\right\}_{n=0}^{\infty}$, $S_{2}: K_{r} \rightarrow K_{r}$ is a nearly uniformly $L_{2}$-Lipschitzian mapping with the sequence $\left\{c_{n}\right\}_{n=0}^{\infty}$, and $\mathcal{Q}$ is a self-mapping of $K_{r} \times K_{r}$, defined by (4.2) such that $\operatorname{Fix}(\mathcal{Q}) \cap \operatorname{SGNVI}\left(K_{r}, T_{i}, g_{i}, i=\right.$ $1,2) \neq \emptyset$. Further, let for each $i=1,2, L_{i} \vartheta<1$, where $\vartheta$ is the same as in (3.16). Then the iterative sequence $\left\{\left(x_{n}, y_{n}\right)\right\}_{n=0}^{\infty}$ generated by Algorithm 4.2, converges strongly to the only element of $\operatorname{Fix}(\mathcal{Q}) \cap \operatorname{SGNVI}\left(K_{r}, T_{i}, g_{i}, i=1,2\right)$.

Proof In view of Theorem 3.2, SGNVI (3.1) has a unique solution $\left(x^{*}, y^{*}\right) \in K_{r} \times K_{r}$. Since $\rho<\frac{r^{\prime}}{1+\left\|T_{1}\left(y^{*}, x^{*}\right)\right\|}$ and $\eta<\frac{r^{\prime}}{1+\left\|T_{2}\left(x^{*}, y^{*}\right)\right\|}$, for some $r^{\prime} \in(0, r)$, it follows from Lemma 3.1 that $\left(x^{*}, y^{*}\right)$ satisfies equations (3.5). Since $\operatorname{SGNVI}\left(K_{r}, T_{i}, g_{i}, i=1,2\right)$ is a singleton set and $\operatorname{Fix}(Q) \cap \operatorname{SGNVI}\left(K_{r}, T_{i}, g_{i}, i=1,2\right) \neq \emptyset$, we deduce that $x^{*} \in \operatorname{Fix}\left(S_{1}\right)$ and $y^{*} \in \operatorname{Fix}\left(S_{2}\right)$. Hence, for each $n \in \mathbb{N} \cup\{0\}$, we can write

$$
\left\{\begin{array}{l}
x^{*}=\left(1-\alpha_{n}\right) x^{*}+\alpha_{n} S_{1}^{n} P_{K_{r}}\left(g_{1}\left(y^{*}\right)-\rho T_{1}\left(y^{*}, x^{*}\right)\right), \\
y^{*}=\left(1-\alpha_{n}\right) y^{*}+\alpha_{n} S_{2}^{n} P_{K_{r}}\left(g_{2}\left(x^{*}\right)-\eta T_{2}\left(x^{*}, y^{*}\right)\right),
\end{array}\right.
$$

where the sequence $\left\{\alpha_{n}\right\}$ is the same as in Algorithm 4.2. Since $g_{1}\left(y^{*}\right), g_{1}\left(y_{n}\right) \in K_{r}, \rho<$ $\frac{r^{\prime}}{1+\left\|T_{1}\left(v^{*}, x^{*}\right)\right\|}$ and $\rho<\frac{r^{\prime}}{1+\left\|T_{1}\left(y_{n}, x_{n}\right)\right\|}$, for some $r^{\prime} \in(0, r)$ and for all $n \in \mathbb{N}$, we can easily check that the points $g_{1}\left(y^{*}\right)-\rho T_{1}\left(y^{*}, x^{*}\right)$ and $g_{1}\left(y_{n}\right)-\rho T_{1}\left(y_{n}, x_{n}\right)(n \in \mathbb{N} \cup\{0\})$, belong to $U\left(r^{\prime}\right)$. 
By using (4.4), (4.5) and Proposition 2.4, we have

$$
\begin{aligned}
\left\|x_{n+1}-x^{*}\right\| & \\
\leq & \left(1-\alpha_{n}\right)\left\|x_{n}-x^{*}\right\|+\alpha_{n}\left\|S_{1}^{n} P_{K_{r}}\left(g_{1}\left(y_{n}\right)-\rho T_{1}\left(y_{n}, x_{n}\right)\right)-S_{1}^{n} P_{K_{r}}\left(g_{1}\left(y^{*}\right)-\rho T_{1}\left(y^{*}, x^{*}\right)\right)\right\| \\
\leq & \left(1-\alpha_{n}\right)\left\|x_{n}-x^{*}\right\| \\
& \quad+\alpha_{n} L_{1}\left(\left\|P_{K_{r}}\left(g_{1}\left(y_{n}\right)-\rho T_{1}\left(y_{n}, x_{n}\right)\right)-P_{K_{r}}\left(g_{1}\left(y^{*}\right)-\rho T_{1}\left(y^{*}, x^{*}\right)\right)\right\|+b_{n}\right) \\
\leq & \left(1-\alpha_{n}\right)\left\|x_{n}-x^{*}\right\| \\
& \quad+\alpha_{n} L_{1}\left(\frac{r}{r-r^{\prime}}\left\|g_{1}\left(y_{n}\right)-g_{1}\left(y^{*}\right)-\rho\left(T_{1}\left(y_{n}, x_{n}\right)-T_{1}\left(y^{*}, x^{*}\right)\right)\right\|+b_{n}\right) \\
& \quad\left(1-\alpha_{n}\right)\left\|x_{n}-x^{*}\right\|+\alpha_{n} L_{1}\left(\frac { r } { r - r ^ { \prime } } \left(\left\|y_{n}-y^{*}-\left(g_{1}\left(y_{n}\right)-g_{1}\left(y^{*}\right)\right)\right\|\right.\right. \\
& \left.\left.+\left\|y_{n}-y^{*}-\rho\left(T_{1}\left(y_{n}, x_{n}\right)-T_{1}\left(y^{*}, x^{*}\right)\right)\right\|\right)+b_{n}\right) .
\end{aligned}
$$

Since $T_{1}$ is $\xi_{1}$-strongly monotone and $v_{1}$-Lipschitz continuous in the first variable, $g_{1}$ is $\kappa_{1}$-strongly monotone and $\theta_{1}$-Lipschitz continuous, in a similar way to the proofs of (3.11) and (3.12), we can get

$$
\begin{aligned}
& \left\|y_{n}-y^{*}-\left(g_{1}\left(y_{n}\right)-g_{1}\left(y^{*}\right)\right)\right\| \leq \sqrt{1-2 \kappa_{1}+\theta_{1}^{2}}\left\|y_{n}-y^{*}\right\|, \\
& \left\|y_{n}-y^{*}-\rho\left(T_{1}\left(y_{n}, x_{n}\right)-T_{1}\left(y^{*}, x^{*}\right)\right)\right\| \leq \sqrt{1-2 \rho \xi_{1}+\rho^{2} v_{1}^{2}}\left\|y_{n}-y^{*}\right\| .
\end{aligned}
$$

By combining (4.6) and (4.7), we obtain

$$
\left\|x_{n+1}-x^{*}\right\| \leq\left(1-\alpha_{n}\right)\left\|x_{n}-x^{*}\right\|+\alpha_{n} L_{1}\left(\psi\left\|y_{n}-y^{*}\right\|+b_{n}\right)
$$

where $\psi$ is the same as in (3.15). Like in the proofs of (4.6)-(4.8), one can prove that

$$
\left\|y_{n+1}-y^{*}\right\| \leq\left(1-\alpha_{n}\right)\left\|y_{n}-y^{*}\right\|+\alpha_{n} L_{2}\left(\varphi\left\|x_{n}-x^{*}\right\|+c_{n}\right),
$$

where $\varphi$ is the same as in (3.15). Letting $L=\max \left\{L_{1}, L_{2}\right\}$, it follows from (4.8) and (4.9) that

$$
\begin{aligned}
& \left\|\left(x_{n+1}, y_{n+1}\right)-\left(x^{*}, y^{*}\right)\right\|_{*} \\
& \quad \leq\left(1-\alpha_{n}\right)\left\|\left(x_{n}, y_{n}\right)-\left(x^{*}, y^{*}\right)\right\|_{*}+\alpha_{n} L\left(\vartheta\left\|\left(x_{n}, y_{n}\right)-\left(x^{*}, y^{*}\right)\right\|_{*}+b_{n}+c_{n}\right) \\
& \quad \leq\left(1-\alpha_{n}(1-L \vartheta)\right)\left\|\left(x_{n}, y_{n}\right)-\left(x^{*}, y^{*}\right)\right\|_{*}+\alpha_{n}(1-L \vartheta) \frac{L\left(b_{n}+c_{n}\right)}{1-L \vartheta},
\end{aligned}
$$

where $\vartheta$ is the same as in (3.16). Since $L \vartheta<1, \sum_{n=1}^{\infty} \alpha_{n}=\infty$ and $\lim _{n \rightarrow \infty} b_{n}=\lim _{n \rightarrow \infty} c_{n}=$ 0 , we note that all the conditions of Lemma 4.4 are satisfied, and so, Lemma 4.4 and (4.10) imply that $\left(x_{n}, y_{n}\right) \rightarrow\left(x^{*}, y^{*}\right)$ as $n \rightarrow \infty$. Hence, the sequence $\left\{\left(x_{n}, y_{n}\right)\right\}_{n=0}^{\infty}$, generated by Algorithm 4.2, converges strongly to the unique solution $\left(x^{*}, y^{*}\right)$ of SGNVI (3.1), that is, the only element of $\operatorname{Fix}(\mathcal{Q}) \cap \operatorname{SGNVI}\left(K_{r}, T_{i}, g_{i}, i=1,2\right)$. This completes the proof. 
Theorem 4.6 Suppose that $T_{i}, g_{i}(i=1,2), \rho$ and $\eta$ are the same as in Theorem 3.2, and let all the conditions of Theorem 3.2 hold. Then the iterative sequence $\left\{\left(x_{n}, y_{n}\right)\right\}_{n=0}^{\infty}$, generated by Algorithm 4.3, converges strongly to the unique solution $\left(x^{*}, y^{*}\right)$ of SGNVI (3.1).

Remark 4.7 Equality (4.3) can be written as follows:

$$
\left\{\begin{array}{l}
z=g_{1}\left(y^{*}\right)-\rho T_{1}\left(y^{*}, x^{*}\right), \\
t=g_{2}\left(x^{*}\right)-\eta T_{2}\left(x^{*}, y^{*}\right), \\
x^{*}=S_{1}^{n} P_{K_{r}}(z), \\
y^{*}=S_{2}^{n} P_{K_{r}}(t),
\end{array}\right.
$$

where $n \geq 0$.

The fixed point formulation (4.11) enables us to suggest the following iterative algorithm for finding an element of the set of fixed points of the nearly uniformly Lipschitzian mapping $\mathcal{Q}=\left(S_{1}, S_{2}\right)$, which is the unique solution of SGNVI (3.1).

Algorithm 4.8 Let $T_{i}, g_{i}(i=1,2), \rho$ and $\eta$ be the same as in SGNVI (3.1). Suppose further that the constants $\rho$ and $\eta$ satisfy condition (3.6) for some $r^{\prime} \in(0, r)$. For a given $\left(z_{0}, t_{0}\right) \in$ $U\left(r^{\prime}\right) \times U\left(r^{\prime}\right)$, compute the iterative sequence $\left\{\left(x_{n}, y_{n}\right)\right\}_{n=0}^{\infty}$ in $K_{r} \times K_{r}$ in the following way:

$$
\left\{\begin{array}{l}
x_{n}=S_{1}^{n} P_{K_{r}}\left(z_{n}\right), \quad y_{n}=S_{2}^{n} P_{K_{r}}\left(t_{n}\right) \\
z_{n+1}=g_{1}\left(y_{n}\right)-\rho T_{1}\left(y_{n}, x_{n}\right) \\
t_{n+1}=g_{2}\left(x_{n}\right)-\eta T_{2}\left(x_{n}, y_{n}\right)
\end{array}\right.
$$

where $S_{i}(i=1,2)$ and $\left\{\alpha_{n}\right\}_{n=0}^{\infty}$ are the same as in Algorithm 4.2.

If for each $i=1,2, S_{i} \equiv I$, then Algorithm 4.8 reduces to the following algorithm.

Algorithm 4.9 Let $T_{i}, g_{i}(i=1,2), \rho$ and $\eta$ be the same as in SGNVI (3.1). Assume further that the constants $\rho$ and $\eta$ satisfy condition (3.6) for some $r^{\prime} \in(0, r)$. For an arbitrary chosen initial point $\left(z_{0}, t_{0}\right) \in U\left(r^{\prime}\right) \times U\left(r^{\prime}\right)$, compute the iterative sequence $\left\{\left(x_{n}, y_{n}\right)\right\}_{n=0}^{\infty}$ in $K_{r} \times K_{r}$ in the following way:

$$
\left\{\begin{array}{l}
x_{n}=P_{K_{r}}\left(z_{n}\right), \quad y_{n}=P_{K_{r}}\left(t_{n}\right), \\
z_{n+1}=g_{1}\left(y_{n}\right)-\rho T_{1}\left(y_{n}, x_{n}\right), \\
t_{n+1}=g_{2}\left(x_{n}\right)-\eta T_{2}\left(x_{n}, y_{n}\right)
\end{array}\right.
$$

where the sequence $\left\{\alpha_{n}\right\}_{n=0}^{\infty}$ is the same as in Algorithm 4.2.

Theorem 4.10 Let $T_{i}, g_{i}(i=1,2), \rho$ and $\eta$ be the same as in Theorem 3.2, and let all the conditions of Theorem 3.2 hold. Assume that $S_{i}(i=1,2)$ and $\mathcal{Q}$ are the same as in Theorem 4.5 such that $\operatorname{Fix}(\mathcal{Q}) \cap \operatorname{SGNVI}\left(K_{r}, T_{i}, g_{i}, i=1,2\right) \neq \emptyset$. Further, let $L_{i} \vartheta<1$ for $i=$ 1,2 , where $\vartheta$ is the same as in (3.16). Then the iterative sequence $\left\{\left(x_{n}, y_{n}\right)\right\}_{n=0}^{\infty}$ generated by Algorithm 4.8, converges strongly to the only element of $\operatorname{Fix}(\mathcal{Q}) \cap \operatorname{SGNVI}\left(K_{r}, T_{i}, g_{i}, i=1,2\right)$. 
Proof In view of Theorem 3.2, SGNVI (3.1) admits a unique solution $\left(x^{*}, y^{*}\right) \in K_{r} \times K_{r}$. It follows from Lemma 3.1 that $x^{*}=P_{K_{r}}\left(g_{1}\left(y^{*}\right)-\rho T_{1}\left(y^{*}, x^{*}\right)\right)$ and $y^{*}=P_{K_{r}}\left(g_{2}\left(x^{*}\right)-\eta T_{2}\left(x^{*}, y^{*}\right)\right)$. Since $\operatorname{SGNVI}\left(K_{r}, T_{i}, g_{i}, i=1,2\right)$ is a singleton set and $\operatorname{Fix}(\mathcal{Q}) \cap \operatorname{SGNVI}\left(K_{r}, T_{i}, g_{i}, i=1,2\right) \neq \emptyset$, we deduce that $x^{*} \in \operatorname{Fix}\left(S_{1}\right)$ and $y^{*} \in \operatorname{Fix}\left(S_{2}\right)$. Accordingly, in view of Remark 4.7, for each $n \geq 0$, we can write

$$
\left\{\begin{array}{l}
x^{*}=S_{1}^{n} P_{K_{r}}(z), \quad y^{*}=S_{2}^{n} P_{K_{r}}(t), \\
z=g_{1}\left(y^{*}\right)-\rho T_{1}\left(y^{*}, x^{*}\right), \\
t=g_{2}\left(x^{*}\right)-\eta T_{2}\left(x^{*}, y^{*}\right) .
\end{array}\right.
$$

From (4.12), (4.13) and the assumptions, it follows that

$$
\begin{aligned}
\left\|z_{n+1}-z\right\|= & \left\|g_{1}\left(y_{n}\right)-g_{1}\left(y^{*}\right)-\rho\left(T_{1}\left(y_{n}, x_{n}\right)-T_{1}\left(y^{*}, x^{*}\right)\right)\right\| \\
\leq & \left\|y_{n}-y^{*}-\left(g_{1}\left(y_{n}\right)-g_{1}\left(y^{*}\right)\right)\right\| \\
& +\left\|y_{n}-y^{*}-\rho\left(T_{1}\left(y_{n}, x_{n}\right)-T_{1}\left(y^{*}, x^{*}\right)\right)\right\| .
\end{aligned}
$$

Since $g_{1}$ is $\kappa_{1}$-strongly monotone and $\theta_{1}$-Lipschitz continuous, and $T_{1}$ is $\xi_{1}$-strongly monotone and $v_{1}$-Lipschitz continuous in the first variable, similar to the proofs of (3.11) and (3.12), one can prove that

$$
\left\|y_{n}-y^{*}-\left(g_{1}\left(y_{n}\right)-g_{1}\left(y^{*}\right)\right)\right\| \leq \sqrt{1-2 \kappa_{1}+\theta_{1}^{2}}\left\|y_{n}-y^{*}\right\|
$$

and

$$
\left\|y_{n}-y^{*}-\rho\left(T_{1}\left(y_{n}, x_{n}\right)-T_{1}\left(y^{*}, x^{*}\right)\right)\right\| \leq \sqrt{1-2 \rho \xi_{1}+\rho^{2} v_{1}^{2}}\left\|y_{n}-y^{*}\right\| .
$$

By combining (4.14)-(4.16), we get

$$
\left\|z_{n+1}-z\right\| \leq\left(\sqrt{1-2 \kappa_{1}+\theta_{1}^{2}}+\sqrt{1-2 \rho \xi_{1}+\rho^{2} v_{1}^{2}}\right)\left\|y_{n}-y^{*}\right\| .
$$

By using (4.12), (4.13) and Proposition 2.4, we have

$$
\begin{aligned}
\left\|y_{n}-y^{*}\right\| & =\left\|S_{2}^{n} P_{K_{r}}\left(t_{n}\right)-S_{2}^{n} P_{K_{r}}(t)\right\| \leq L_{2}\left(\left\|P_{K_{r}}\left(t_{n}\right)-P_{K_{r}}(t)\right\|+c_{n}\right) \\
& \leq L_{2}\left(\frac{r}{r-r^{\prime}}\left\|t_{n}-t\right\|+c_{n}\right) .
\end{aligned}
$$

Substituting (4.18) in (4.17), we get

$$
\left\|z_{n+1}-z\right\| \leq L_{2} \psi\left\|t_{n}-t\right\|+L_{2} \psi c_{n}
$$

where $\psi$ is the same as in (3.15). Like in the proofs of (4.14)-(4.19), one can establish that

$$
\left\|t_{n+1}-t\right\| \leq L_{1} \varphi\left\|z_{n}-z\right\|+L_{1} \varphi b_{n}
$$


where $\varphi$ is the same as in (3.15). Let $L=\max \left\{L_{i}: i=1,2\right\}$. Then, applying (4.19) and (4.20), we obtain

$$
\begin{aligned}
\left\|\left(z_{n+1}, t_{n+1}\right)-(z, t)\right\|_{*} & \leq L \vartheta\left\|\left(z_{n}, t_{n}\right)-(z, t)\right\|_{*}+L \vartheta\left(b_{n}+c_{n}\right) \\
& \leq(L \vartheta)^{2}\left\|\left(z_{n-1}, t_{n-1}\right)-(z, t)\right\|+(L \vartheta)^{2}\left(b_{n-1}+c_{n-1}\right)+L \vartheta\left(b_{n}+c_{n}\right) \\
& \leq \cdots \leq(L \vartheta)^{n+1}\left\|\left(z_{0}, t_{0}\right)-(z, t)\right\|+\sum_{i=0}^{n}(L \vartheta)^{i+1}\left(b_{n-i}+c_{n-i}\right), \quad
\end{aligned}
$$

where $\vartheta$ is the same as in (3.16). Since $L \vartheta<1$ and $\lim _{n \rightarrow \infty} b_{n}=\lim _{n \rightarrow \infty} c_{n}=0$, it follows that the right side of the above inequality tends to zero, as $n \rightarrow \infty$ and so $\left(z_{n}, t_{n}\right) \rightarrow(z, t)$, as $n \rightarrow \infty$. By using (4.12), (4.13) and Proposition 2.4, we have

$$
\begin{aligned}
\left\|x_{n}-x^{*}\right\| & =\left\|S_{1}^{n} P_{K_{r}}\left(z_{n}\right)-S_{1}^{n} P_{K_{r}}(z)\right\| \leq L_{1}\left(\left\|P_{K_{r}}\left(z_{n}\right)-P_{K_{r}}(z)\right\|+b_{n}\right) \\
& \leq L_{1}\left(\frac{r}{r-r^{\prime}}\left\|z_{n}-z\right\|+b_{n}\right) .
\end{aligned}
$$

Since $\lim _{n \rightarrow \infty} z_{n}=z, \lim _{n \rightarrow \infty} t_{n}=t$ and $\lim _{n \rightarrow \infty} b_{n}=\lim _{n \rightarrow \infty} c_{n}=0$, from inequalities (4.18) and (4.22), it follows that $y_{n} \rightarrow y^{*}, x_{n} \rightarrow x^{*}$, as $n \rightarrow \infty$. Hence, the sequence $\left\{\left(x_{n}, y_{n}\right)\right\}_{n=0}^{\infty}$, generated by Algorithm 4.8, converges strongly to the unique solution $\left(x^{*}, y^{*}\right)$ of system (3.1), that is, the only element of $\operatorname{Fix}(\mathcal{Q}) \cap \operatorname{SGNVI}\left(K_{r}, T_{i}, g_{i}, i=1,2\right)$. This completes the proof.

Theorem 4.11 Let $T_{i}, g_{i}(i=1,2), \rho$ and $\eta$ be the same as in Theorem 3.2, and let all the conditions of Theorem 3.2 hold. Then the iterative sequence $\left\{\left(x_{n}, y_{n}\right)\right\}_{n=0}^{\infty}$, generated by Algorithm 4.9, converges strongly to the unique solution $\left(x^{*}, y^{*}\right)$ of SGNVI (3.1).

\section{Competing interests}

The authors declare that they have no competing interests.

\section{Authors' contributions}

All authors contributed equally and significantly in this paper. All authors read and approved the final manuscript.

\section{Author details}

'Department of Mathematics, Faculty of Science, Naresuan University, Phitsanulok, 65000, Thailand. ${ }^{2}$ Department of Mathematics, Sari Branch, Islamic Azad University, Sari, Iran.

\section{Acknowledgements}

The authors would like to thank the referees for the complete reading of the first version of this work and for the pertinent suggestions allowing us to improve the presentation of the paper. The first author is supported by the National Research Council of Thailand (Project No. R2555B025).

Received: 10 November 2012 Accepted: 25 July 2013 Published: 9 August 2013

\section{References}

1. Stampacchia, G: Formes bilineaires coercitives sur les ensembles convexes. C. R. Acad. Sci. Paris 258, 4413-4416 (1964)

2. Bensoussan, A, Lions, JL: Application des Inéquations Variationelles en Control et en Stochastiques. Dunod, Paris (1978)

3. Glowinski, R, Letallec, P: Augmented Lagrangian and Operator-Splitting Methods in Nonlinear Mechanics. SIAM Stud. Appl. Math. SIAM, Philadelphia (1989)

4. Harker, PT, Pang, JS: Finite-dimensional variational inequality and nonlinear complementarity problems: a survey of theory, algorithm and applications. Math. Program. 48, 161-220 (1990)

5. Lions, JL, Stampacchia, G: Variational inequalities. Commun. Pure Appl. Math. 20, 493-512 (1967)

6. Verma, RU: Generalized system for relaxed cocoercive variational inequalities and projection methods. J. Optim. Theory Appl. 121, 203-210 (2004) 
7. Chang, SS, Joseph Lee, HW, Chan, CK: Generalized system for relaxed cocoercive variational inequalities in Hilbert spaces. Appl. Math. Lett. 20, 329-334 (2007)

8. Huang, Z, Noor, MA: An explicit projection method for a system of nonlinear variational inequalities with different $(\gamma, r)$-cocoersive mappings. Appl. Math. Comput. 190, 356-361 (2007)

9. Bounkhel, M, Tadji, L, Hamdi, A: Iterative schemes to solve nonconvex variational problems. J. Inequal. Pure Appl. Math. 4, 1-14 (2003)

10. Clarke, FH, Ledyaev, YS, Stern, RJ, Wolenski, PR: Nonsmooth Analysis and Control Theory. Springer, New York (1998)

11. Clarke, FH, Stern, RJ, Wolenski, PR: Proximal smoothness and the lower $C^{2}$ property. J. Convex Anal. 2, 117-144 (1995)

12. Poliquin, RA, Rockafellar, RT, Thibault, L: Local differentiability of distance functions. Trans. Am. Math. Soc. 352, 5231-5249 (2000)

13. Balooee, J, Cho, YJ, Kang, MK: The Wiener-Hopf equation technique for solving general nonlinear regularized nonconvex variational inequalities. Fixed Point Theory Appl. 2011, 87 (2011). doi:10.1186/1687-1812-2011-86

14. Noor, MA, Petrot, N, Suwannawit, J: Existence theorems for multivalued variational inequality problems on uniformly prox-regular sets. Optim. Lett. (2012). doi:10.1007/s11590-012-0545-x

15. Pang, LP, Shen, J, Song, HS: A modified predictor-corrector algorithm for solving nonconvex generalized variational inequalities. Comput. Math. Appl. 54, 319-325 (2007)

16. Petrot, N: Some existence theorems for nonconvex variational inequalities problems. Abstr. Appl. Anal. 2010, Article ID 472760 (2010). doi:10.1155/2010/472760

17. Suwannawit, J, Petrot, $\mathrm{N}$ : Existence theorems for quasivariational inequality problem on proximally smooth sets. Abstr. Appl. Anal. 2013, Article ID 612819 (2013)

18. Noor, MA, Huang, Z: Three-step iterative methods for nonexpansive mappings and variational inequalities. Appl. Math. Comput. 187, 680-685 (2007)

19. Sahu, DR: Fixed points of demicontinuous nearly Lipschitzian mappings in Banach spaces. Comment. Math. Univ. Carol. 46, 653-666 (2005)

20. Balooee, J, Cho, YJ: Algorithms for solutions of extended general mixed variational inequalities and fixed points. Optim. Lett. (2012). doi:10.1007/s11590-012-0516-2

21. Balooee, J, Petrot, N: Algorithms for solving system of extended general variational inclusions and fixed points problems. Abstr. Appl. Anal. 2012, Article ID 569592 (2012)

22. Clarke, FH: Optimization and Nonsmooth Analysis. Wiley, New York (1983)

23. Bounkhel, M: Existence results of nonconvex differential inclusions. Port. Math. 59, 283-309 (2002)

24. Bounkhel, M: General existence results for second order nonconvex sweeping process with unbounded perturbations. Port. Math. 60(3), 269-304 (2003)

25. Bounkhel, M, Azzam, L: Existence results on the second order nonconvex sweeping processes with perturbations. Set-Valued Anal. 12(3), 291-318 (2004)

26. Bounkhel, $\mathrm{M}$, Thibault, L: Further characterizations of regular sets in Hilbert spaces and their applications to nonconvex sweeping process. Preprint, Centro de Modelamiento Matematico (CMM), Universidad de Chile (2000)

27. Canino, A: On p-convex sets and geodesics. J. Differ. Equ. 75, 118-157 (1988)

28. Moudafi, A: Projection methods for a system of nonconvex variational inequalities. Nonlinear Anal. 71, 517-520 (2009)

29. Verma, RU: Projection methods, algorithms, and a new system of nonlinear variational inequalities. Comput. Math. Appl. 41, 1025-1031 (2001)

30. Verma, RU: General convergence analysis for two-step projection methods and applications to variational problems. Appl. Math. Lett. 18, 1286-1292 (2005)

31. Goebel, K, Kirk, WA: A fixed point theorem for asymptotically nonexpansive mappings. Proc. Am. Math. Soc. 35, 171-174 (1972)

32. Kirk, WA, Xu, HK: Asymptotic pointwise contractions. Nonlinear Anal. 69, 4706-4712 (2008)

33. Weng, XL: Fixed point iteration for local strictly pseudo-contractive mapping. Proc. Am. Math. Soc. 113, 727-732 (1991)

doi:10.1186/1029-242X-2013-377

Cite this article as: Petrot and Balooee: General nonconvex variational inclusions and fixed point problems. Journal of Inequalities and Applications 2013 2013:377.

\section{Submit your manuscript to a SpringerOpen ${ }^{\circ}$ journal and benefit from:}

- Convenient online submission

Rigorous peer review

- Immediate publication on acceptance

- Open access: articles freely available online

- High visibility within the field

- Retaining the copyright to your article 